\section{Visible persistence is the result of spatiotemporal filtering: A reply to Stelmach and Hearty}

\author{
JOYCE E. FARRELL and EDWARD J. KESSLER \\ Stanford University, Stanford, California
}

Continuous motion is commonly represented on video displays and film by a succession of discrete samples. As the temporal sampling of the continuous motion is increased, the appearance of the discrete samples becomes quite similar to the appearance of continuous motion (Sperling, 1976). When the continuous motion is not sampled at a high enough rate, the appearance of continuous motion is destroyed (Watson, Ahumada, \& Farrell, 1983). In this case, we say the image motion has been undersampled. Depending on the nature of the motion and the undersampling, the appearance of the discrete samples can take on a variety of forms.

One form of undersampling that destroys the appearance of continuous motion occurs when a line is moving rapidly but the sampling rate is slightly lower than required to accurately represent the line's appearance. This undersampling results in the appearance of a set of lines that are present simultaneously at the spatial locations where the continuously moving line was sampled. Farrell (1984) measured the stimulus parameters at which this kind of undersampling occurs as well as some of the perceptual consequences of this form of undersampling. By asking the observers to report how many lines they saw on the screen simultaneously, she obtained a measure of the visible persistence of the first line. Farrell varied the angular distance between samples, $\delta x$, and the time between samples, $\delta t$. She concluded that when measured as part of a sequence of discrete samples, the visible persistence of the first sample was proportional to the angular distance separating the sample from samples occurring later in time. She noted that if the visible persistence to the first sample reflected the activity of a neural substrate, then that activity was reduced in duration by the spatially and temporally adjacent samples that followed.

Stelmach and Hearty (1988) replotted Farrell's data and found that the number of successively presented samples that appeared to be simultaneously visible was proportional to the velocity of the time-sampled moving line. This was another way of stating Farrell's observation that visible persistence is proportional to the distance between samples. For example, let $N$ be the number of samples perceived simultaneously, let $p$ be the visible persistence of the first sample. The persistence hypothesis described

The authors would like to thank Professor Brian A. Wandell for helpful discussions and critique of this manuscript. E. J. Kessler is now at Valid Logic, 2820 Orchard Parkway, San Jose, CA 95134. Requests for reprints should be addressed to J. E. Farrell, Hewlett-Packard Corporate Engineering, P. O. Box 10350, Palo Alto, CA 94303-0867. in Farrell (1984; see also Allport, 1968, 1970; Efron \& Lee, 1971) predicts that the number of stimuli perceived simultaneously is a function of the duration of visible persistence $N=p / \delta t$, where $p$ is the duration of visible persistence. Farrell (1984) found that the duration of visible persistence was a linear function of the sample spacing, $p=b \delta x+a$. Since observers always see at least one line, it follows that $N=\max [(b \delta x+a) / \delta t, 1]$. If $a /(\delta t)$ is small compared to one, $N$ is proportional to angular velocity for values above one. Stelmach and Hearty (1988) argue that expressing the relationship with respect to "the principal conjugate"--by which they mean angular velocityis simpler and in some way would "agree with the current trend toward the use of motion and velocity tuning as the basis for mechanisms in psychophysical, computational, and neurophysiological modeling" (p. 199). We respond to Stelmach and Hearty's comments by illustrating how linear spatiotemporal mechanisms that have been proposed for motion detection can produce results such as those reported by Farrell (1984).

Several models of spatial vision (Ahumada \& Watson, 1985; Watson, 1983; Wilson \& Gelb, 1984) and motion perception (Adelson \& Bergen, 1985; van Santen \& Sperling, 1985; Watson \& Ahumada, 1985) incorporate the assumption that early visual processing is mediated by locally linear units with separable spatial and temporal impulse responses. This simplifying assumption is supported by the important work of Enroth-Cugell and Robson $(1966,1984)$, which indicates that retinal ganglion cells are locally linear with respect to contrast. In many computational models of human vision, the center-surround organization of retinal ganglion cells is modeled by a spatial weighting function, as illustrated in Figure 1A, and the temporal response of retinal units is modeled by a temporal impulse response function, as illustrated in Figure $1 \mathrm{~B}$. The response of locally linear retinal units to any spatiotemporal stimulus is predicted by the convolution of the stimulus with the unit's spatial and temporal impulse response functions.

The response of a retinal unit to a single sample depends on both the location of the sample within the unit's receptive field and the temporal impulse response of the unit. The spatial weighting function determines the initial amplitude of the unit's response to a point of light falling within its receptive field (Figure 1A). The temporal impulse response function determines how the unit's response decays over time (Figure 1B). When a sample falls on some portion of the receptive field, the temporal response is scaled by a factor that depends upon the location of the sample in the receptive field. The sum over time of all such scaled temporal impulses is the convolution of the stimulus with the spatial and temporal impulse response functions of the linear unit.

The response of a linear unit to a series of samples is simply the sum of individual responses to each sample. 


\section{A: spatial weighting function}

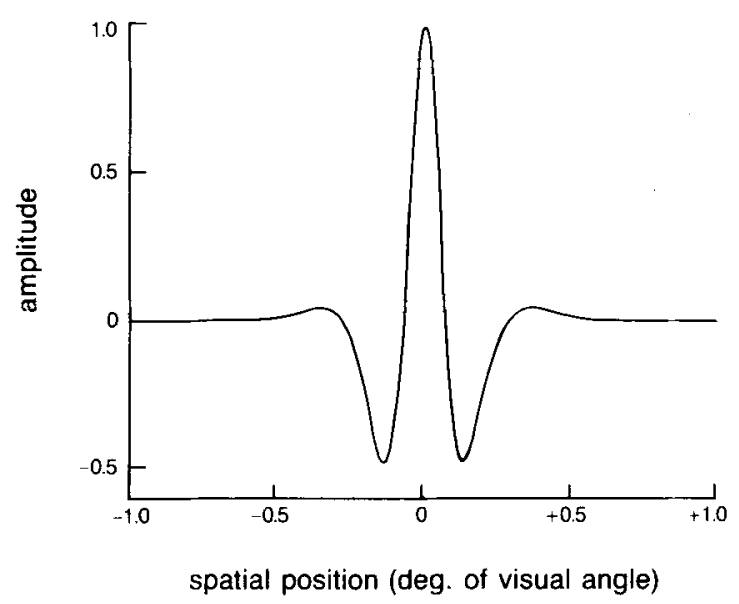

B: temporal impulse response function

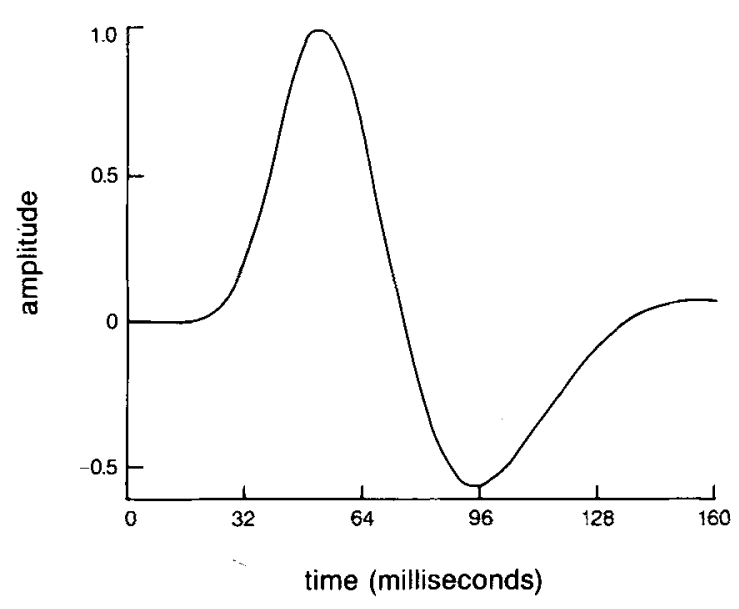

Figure 1. One-dimensional spatial weighting function and temporal impulse response function of a hypothetical retinal unit. (A) A spatial weighting function with peak spatial sensitivity at 2.8 cycles per degree of visual angle (derived from Wilson \& Gelb, 1984). (B) A temporal impulse response function for an adaptation level of $7.5 \mathrm{Tr}$ (derived from Kelly, 1971).

Figure 2 shows the unit's response to (A) a sample presented in the center of the unit's spatial weighting function, (B) a sample presented in the unit's surround, and (C) the successive presentation of the two samples. Figure 3 illustrates the fact that the overall response of a linear unit will depend on the distance between the successive samples. As the distance between the successive samples decreases, more stimuli will fall in the surround of a given unit and thus influence the overall temporal response of that unit. We would like to identify the overall response of such a unit with the visible persistence at a location. As we have just shown, the overall response of a single unit depends upon the spatial properties of the stimuli that fall in its receptive field.

Our explanation of the duration of visible persistence requires an additional assumption that the response of linear retinal units is followed by a nonlinear threshold.

A.

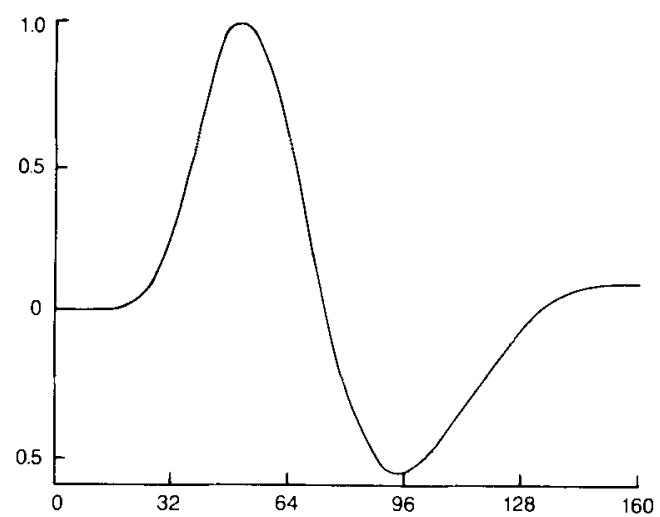

B.

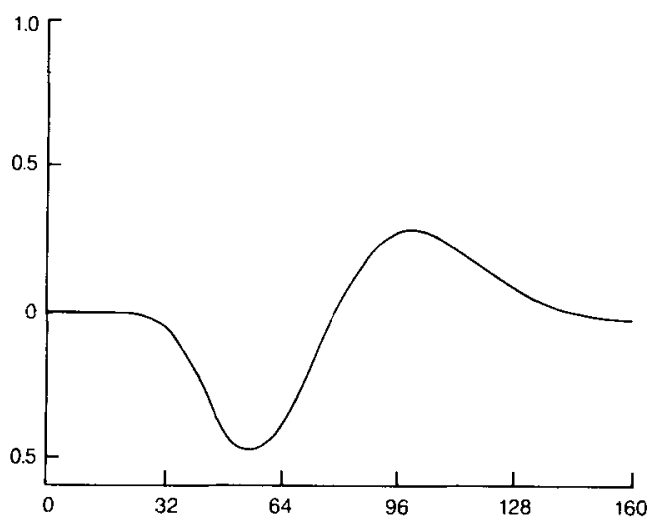

c.

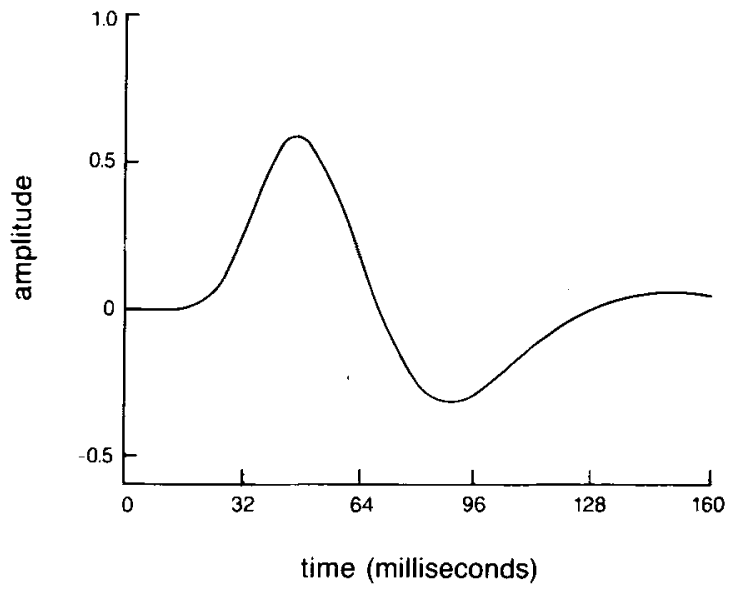

Figure 2. (A) The temporal response to one sample of a moving light taken at time $t=0$ msec and position $x=0^{\circ}$ (see Figure 1A). (B) The temporal response to one sample of a moving light taken at time $t=5 \mathrm{msec}$ and position $x=0.15^{\circ}$. (C) The overall temporal response to the two samples of a moving light taken at time $t=0$ msec, position $x=0^{\circ}$, and at time $t=5 \mathrm{msec}$, position $\boldsymbol{x}=\mathbf{0 . 1 5 ^ { \circ }}$. 


\section{overall temporal response}

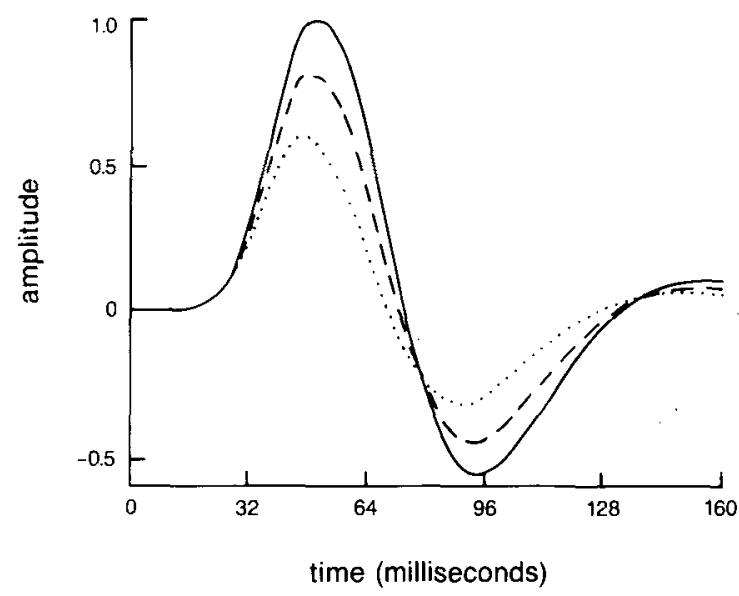

Figure 3. The overall temporal response to two samples of a moving light taken at time $t=0$ msec and time $t=5 \mathrm{msec}$. The first sample is always located at position $x=0^{\circ}$. The location of the second sample varies with the velocity of the moving light. The solid line depicts the response to two samples of a light moving at a relatively fast velocity such that the position of the second sample is at $x=0.28^{\circ}$. The dashed line depicts the response to two samples separated by $x=0.20^{\circ}$. The dotted line depicts the response to two samples separated by $x=0.15^{\circ}$.

Once the response of a single unit is above a fixed threshold, we will consider a sample to be visible at the location corresponding to the center of the unit's spatial weighting function. Once the response falls below a fixed threshold, we will consider the sample to be no longer visible. The duration of visible persistence corresponds to the length of time the unit's response is above the amplitude threshold for visibility. There will, of course, be threshold assumptions that will not predict the results obtained by Farrell (1984). However, visibility thresholds corresponding to a fixed percentage of the maximum amplitude of the unit's response or the absolute value of a fixed amplitude will predict the pattern of results reported by Farrell. For example, Figure 3 shows that the amount of time the response of a single unit is greater than a fixed threshold decreases with the distance between successive samples. The overall response is shortened because of stimulation of the spatial surround.

Simple linear units with separable spatial and temporal impulse responses constitute the basic components of many computational models for spatial vision (Ahumada \& Watson, 1985; Watson, 1983; Wilson \& Gelb, 1984) and motion perception (Adelson \& Bergen, 1985; van Santen \& Sperling, 1985; Watson \& Ahumada, 1985). We are currently exploring which forms of undersampling and visible persistence can be modeled using simple linear components and simple assumptions about visibility thresholds.

\section{REFERENCES}

Adeıson, E. H. \& BERGEN, J. R. (1985). Spatiotemporal energy models for the perception of motion. Journal of the Optical Society of America, 2, 284-299.

Ahumada, A. J., Jr. \& Watson, A. B. (1985). Equivalent noise model for contrast detection and discrimination. Journal of the Optical Society of America, 2, 1133-1139.

Allport, D. A. (1968). Phenomenal simultaneity and the perceptual moment hypothesis. British Journal of Psychology, 59, 395-406.

Allport, D. A. (1970). Temporal summation and phenomenal simultaneity: Experiments with the radius display. Quarterly Journal of Experimental Psychology, 22, 686-701.

EFroN, F., \& LEE, D. N. (1971). The visual persistence of a moving stroboscopically illuminated object. American Joumal of Experimental Psychology, 84, 365-375.

Enroth-Cugell, C., \& Robson, J. G. (1966). The contrast sensitivity of retinal ganglion cells of the cat. Journal of Physiology, 197, 551-566.

Enroth-Cugell, C., \& Robson, J. G. (1984). Functional characteristics and diversity of cat retinal ganglion cells. Investigative Ophthalmol ogy \& Visual Science, 25, 250-267.

FARRELL, J. E. (1984). The visible persistence of moving objects. Journal of Experimental Psychology: Human Perception \& Performance, 10, 502-511.

KeLLY, D. H. (1971). Theory of flicker and transient responses: I. Uniform fields. Journal of the Optical Society of America, 61, 537-546.

SPERLING, G. (1976). Movement perception in computer-driven dis plays. Behavior Research Methods \& Instrumentation, 8, 144-151.

Stelmach, L. B., \& Hearty, P. J. (1988). Velocity or spatial and temporal separation: A comment on Farrell. Perception \& Psychophysics, 43, 199-200.

van Santen, J. P. H., \& Sperling, G. (1985). Elaborated Reichardt detectors. Journal of the Optical Society of America, 2, 300-321.

WATson, A. B. (1983). Detection and recognition of simple spatial forms. In O. J. Braddick \& A. C. Sleigh (Eds.), Physical and biological processing of images (pp. 100-114). New York: SpringerVerlag.

Watson, A. B., \& Ahumada, A. J., JR. (1985). Model of human visualmotion sensing. Journal of the Optical Society of America, 2, 322-341.

Watson, A. B., Ahumada, A. J., Jr., \& Farrell, J. E. (1983). The window of visibility: A psychophysical theory of fidelity in time-sampled visual motion displays (NASA Technical Paper 2211). Washington, DC: NASA. (Reprinted in the Journal of the Optical Society of America, Vol. 3, No. 3, March 1986)

Wilson, H. R., \& GELB, D. (1984). Modified line-element theory for spatial-frequency and width discrimination. Journal of the Optical Society of America, 1, 124-131.

(Manuscript received September 15, 1987; accepted for publication September 15, 1987.) 